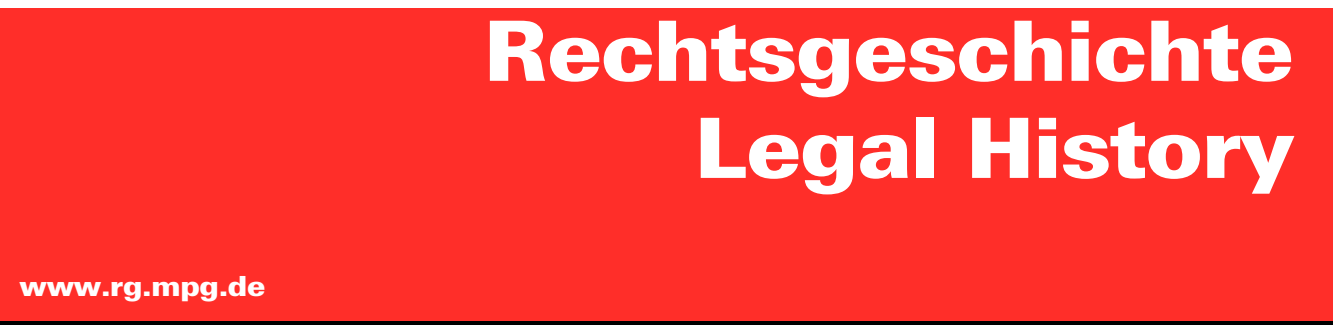

http://www.rg-rechtsgeschichte.de/rg26

$\operatorname{Rg} 262018 \quad 492-496$

Zitiervorschlag: Rechtsgeschichte - Legal History Rg 26 (2018)

http://dx.doi.org/10.12946/rg26/492-496

\title{
Alessandro Somma*
}

\section{Ancora teorie sul totalitarismo!?}

Sulla comparazione tra Germania nazista e Repubblica democratica tedesca

[Still Theories on Totalitarianism!? On the Comparison Between

Nazi-Germany and East Germany]

* Università degli Studi di Ferrara, alessandro.somma@unife.it

Dieser Beitrag steht unter einer Creative Commons cc-by-nc-nd 3.0 
hard Lösener, suchten zwar Freislers Furor mit juristischen Bedenken zu mäßigen, wie sich aus einem Beratungsprotokoll vom 5. Juni 1934 ergibt. Die »Nürnberger Gesetze« waren aber mit der Schaffung eines staatsbürgerlichen Minderstatus für Juden, dem Verbot von »Mischehen«, der Strafbarkeit von "Rassenschande« samt einer langen Reihe weiterer »ausgrenzender« Verordnungen eine nun antisemitische und letztlich tödliche Variante des amerikanischen Modells. Gewiss hätte es für die Nationalsozialisten dieses Modells nicht bedurft, aber da es vorhanden war, wurde es auch genutzt. Die Radikalen hatten sich durchgesetzt, die retardierenden Kräfte um Gürtner trösteten sich damit, die Rassenpolitik nun rechtlich »eingehegt« zu haben.

Eine Woche nach der Verkündung der »Nürnberger Gesetze« startete eine offizielle deutsche Delegation von Juristen zu einer Reise in die USA. Whitman schildert dieses bedrückende Nachspiel mit einer Mischung von Ironie und Abscheu. Die Gruppe, 45 Personen insgesamt, trat mit demonstrativer Zufriedenheit auf, ihre schon lange gehegten Ziele erreicht zu haben. Dass sie im »jüdischen « New York auf heftige Proteste stieß, verdarb einerseits die Stimmung, wurde aber andererseits auch als Bestätigung gedeutet, dass das »Welt- judentum« die Kampfansage des Nationalsozialismus verstanden und angenommen hatte.

Am Ende des Buchs weitet sich Whitmans Blick nochmals. Er erinnert nicht nur an die amerikanische Geschichte seit der Abschaffung der Sklaverei, sondern auch an das britische Kolonialrecht, an die Einwanderungsgesetzgebung Brasiliens, an Südafrika, Australien und andere Beispiele, in denen "von Rechts wegen « Ungleichheiten nach biologischen Kriterien geschaffen worden waren. Das mindert gleichwohl nicht die damals dominante Rolle der USA als ein von den Nationalsozialisten nachweislich benutztes Referenzmodell, noch ändert sich etwas an der Bewertung des Antisemitismus und der brutalen Verfolgung auch anderer Minderheiten durch das NS-Regime. Doch kann die schärfere Beleuchtung des amerikanischen Anteils am Phänomen der Diskriminierung nach Hautfarbe, Religion, Geschlecht, Sprache und anderen Merkmalen sichtbar machen, dass der ganze Spuk nicht etwa mit dem 8. Mai 1945 gebannt war. Wer insoweit jemals Illusionen hegte, wird durch den Blick auf die heutige Weltpolitik und speziell auf die gegenwärtige Regierung der USA eines Schlechteren belehrt.

\title{
Alessandro Somma \\ Ancora teorie sul totalitarismo!?
}

\author{
Sulla comparazione tra Germania nazista e Repubblica democratica tedesca*
}

SCIENZA GiURIdica E DitTATURe - Al principio del nuovo millennio Jan Schröder ha licenziato una storia del sapere giuridico tra il 1500 e il 1850 , divenuta per molti aspetti un classico, con cui ha indagato ciò che renderebbe il diritto una scienza: un metodo e un oggetto specifici. Di qui il filo conduttore dell'opera, concepita come una storia della metodologia giuridica (juristische Methodenlehre), quindi delle teorie circa l'individuazione e l'accertamento del diritto in generale o in riferimento a un caso concreto, e nello stesso tempo una storia della teoria delle fonti (Rechtsquellenlehre), incluse le tecniche utilizzate dall'interprete per fronteggiare antinomie e lacune. Il tutto riferito

* Jan Schröder, Rechtswissenschaft in Diktaturen. Die juristische Methodenlehre im NS-Staat und in der DDR, München: C. H. Beck 2016, XIV, 146 S., ISBN 978-3-406-69606-0 
all'Europa continentale, sebbene con un focus prevalente sull'area tedesca. ${ }^{\mathbf{1}}$

Dopo un decennio, una nuova edizione dell'opera ha ampliato l'orizzonte temporale dell'indagine estendendolo sino al 1933, ovvero sino alla conclusione dell'esperienza weimariana. È l'occasione per inquadrare quanto per Schröder costituisce l'essenza della transizione dall'approccio al diritto tipico della Scuola storica a quello caratteristico dei decenni successivi. Se infatti quest'ultima vedeva nel diritto un'espressione dello spirito popolare, e dunque lo intendeva in senso idealistico oggettivo, nell'epoca successiva è prevalso un approccio volontarista, per cui il diritto rappresenta la volontà di un'entità descritta in termini di maggioranza, comunità o Stato. ${ }^{2}$

Ebbene, la contrapposizione tra concezioni oggettive e concezioni volontariste del diritto costituisce il filo conduttore dell'ultimo lavoro di Schröder, che rappresenta un'integrazione della sua opera con riferimento all'epoca che copre la dittatura nazista e l'esperienza socialista tedesco orientale. Lì si afferma che il regime nazista, esattamente come la Repubblica democratica tedesca, sono stati dittature in quanto governati dalla volontà del Führer o dalle determinazioni del Partito socialista unitario. Di qui la connotazione volontarista del diritto, che conviveva però con concezioni di matrice oggettiva: quelle ispirate dai riferimenti ai principi völkisch o alle leggi del socialismo.

Lo schema era però instabile e produceva pertanto conflitti coinvolgenti la metodologia giuridica e la teoria delle fonti, che si risolvevano a tutto vantaggio dell'approccio volontarista: ricorrendo alla finzione per cui la via viene indicata dal dittatore in quanto interprete autentico dello spirito popolare o depositario della verità scientifica. Il tutto in assenza di spazi lasciati all'integrazione giurisprudenziale del diritto, che comunque non poteva certo fondarsi sulla coscienza del giudice.

Comparazione e ideologia - Il lavoro di Schröder è innanzi tutto un lavoro comparatistico, nel quale si ricorre ai raffronti per ottenere risultati che non si sarebbero altrimenti messi a fuoco con la stessa nitidezza.

Comparare porta naturalmente a classificare, ovvero a raggruppare ordinamenti in base alla ricorrenza di determinate caratteristiche: la produzione di tassonomie è forse il compito principale attribuito ai comparatisti. Tutte le classificazioni sono pienamente legittime, se condotte in modo rigoroso, ma non tutte sono utili, ovvero assolvono al compito di incrementare le nostre conoscenze, o eventualmente di assecondare l'economia del discorso attorno al diritto. Ma tutte sono in qualche modo affette da tensione ideologica o quantomeno ideale: se comparare equivale a giudicare, classificare significa in ultima analisi alimentare una geografia dei corpi politici. ${ }^{3}$

Sono, queste ultime, premesse indispensabili ad affrontare un esercizio comparatistico che ha per oggetto fascismo e socialismo, e in particolare il regime nazista e la Repubblica democratica tedesca. Giacché possiamo oramai dire, sulla scorta di decenni di attività di ricerca condotta soprattutto dagli storici puri, che simili raffronti possono essere utili se pensati per mettere in luce alcuni specifici aspetti: ad esempio il ruolo del partito unico o del capo carismatico. ${ }^{4}$ Non sono invece rilevanti le acquisizioni in altri ambiti, nei quali l'accostamento risulta incapace di incrementare conoscenze, se non addirittura capace di manipolare quelle di cui già disponiamo.

Più precisamente sono utili i raffronti tra totalitarismi se riguardano specifiche modalità o particolari apparati utilizzati per sopprimere le libertà politiche. Peraltro, nel fascismo, ciò è avvenuto per riformare le libertà economiche, ovvero per sostenere e non certo per reprimere i fondamenti del capitalismo: i pubblici poteri hanno sopperito all'incapacità della mano invisibile di assicurare il funzionamento dell'ordine economico, e in tale prospettiva hanno utilizzato la concorrenza come strumento di direzione politica dei comportamenti
1 JAN SCHRÖDER, Geschichte der juristischen Methode vom Humanismus bis zur historischen Schule (15001850), 2. ed., München 2012.

2 Jan Schröder, Recht als Wissenschaft. Geschichte der juristischen Methodenlehre in der Neuzeit (1500-1933), München 2001.

3 Alessandro Somma, La geografia dei corpi politici. Classificazioni e genea- logie tra diritto privato comparato, diritto pubblico comparato e ortodossia neoliberale, in: Diritto pubblico comparato ed europeo 19 (2017) 1217-1249.

4 Per tutti Emilio Gentile, La via italiana al totalitarismo. Il partito e lo Stato nel regime fascista, nuova ed., Roma 2008. Anche Hans-Ulrich Wehler, Deutsche Gesellschaftsge- schichte, vol. 5 (1949-1990), München 2008, 417. 
dei consociati. Tutto l'opposto di quanto ha fatto il socialismo, che ha invece soppresso, oltre alle libertà politiche, anche il sistema delle libertà economiche, con ciò ricalcando uno schema che da questo punto di vista non è certo assimilabile a quello fatto proprio dal fascismo.

Insomma, se si riconducono fascismo e socialismo a un medesimo raggruppamento si devono trascurare le vicende che attengono al modo di essere dell'ordine economico: quelle di matrice teorica, ma anche quelle concernenti l'individuazione dei centri di interesse compromessi con la dittatura. Il che è sovente un risultato voluto, non di rado indicato come la ragione prima per l'elaborazione di una teoria sul totalitarismo, e per il contestuale rigetto delle teorie sul fascismo: quelle che consentono al contrario di approfondire il nesso tra soppressione delle libertà politiche $\mathrm{e}$ riforma delle libertà economiche. ${ }^{5}$

Non si tratta qui di riproporre il mito della incomparabilità dell'Olocausto, concernente il piano della morale piuttosto che quello dell'approfondimento scientifico. Si tratta più semplicemente di mettere in luce, oltre alla relativa inutilità dell'accostamento tra fascismo e socialismo, anche e soprattutto il suo costituire uno strumento di lotta politica: ${ }^{6}$ utilizzato prima per alimentare la Guerra fredda e poi, al crollo del blocco socialista, per consolidare il moto verso la fine della storia intesa come assolutizzazione dell'ortodossia neoliberale. Il che non è certo un caso, dal momento che, nello studio dell'ordine economico, le teorie sul totalitarismo perseguono innanzi tutto il fine di presidiare la normalità capitalistica. ${ }^{7}$ Mirano cioè a impedire che si sviluppino forme di redistribuzione della ricchezza diverse da quelle affidate al principio di concorrenza, ovvero che si metta in discussione l'equazione che identifica l'inclusione sociale con l'inclusione nel mercato.

Continuità PERSONALi E ISTITUZionali - Un libro sulla scienza giuridica è in ultima analisi un libro sui cultori del diritto, sulle loro strategie di legittimazione in quanto ceto: durante la dittatura, ma anche e soprattutto dopo la sua conclusione, quando occorre cancellare le tracce della collaborazione o almeno rileggerle per alterarne il senso. Circostanza peraltro alla portata del giurista, la cui legittimazione è di tipo tecnico professionale, ovvero formalmente incentrata proprio sulle vicende cui Schröder ha dedicato la sua ricerca: sull'individuazione e l'accertamento di ciò che costituisce diritto, piuttosto che sul suo contenuto.

Sappiamo molto bene che i giuristi hanno fornito un notevole contributo all'edificazione della dittatura nazista, esattamente come di quella socialista. Meno documentate sono però le continuità personali che hanno caratterizzato il passaggio dalla dittatura alla democrazia, che sono state numerose e inquietanti. $\mathrm{O}$ meglio, sono state documentate, ma con esiti molto diversi a seconda che sul banco degli imputati si trovasse il nazismo o il socialismo.

Nel primo caso il tutto è avvenuto con notevole ritardo, e soprattutto con il fondamentale contributo dei non giuristi. Giacché i cultori del diritto erano impegnati a disegnare l'identikit del cultore del diritto nazista come sanguinario carnefice prono al volere della dittatura, e di riflesso a negare che fossero compromessi tutti coloro i quali non potevano ritenersi tali. ${ }^{\mathbf{8}}$ Tutto l'opposto di quanto avvenuto con la dittatura socialista, anche perché in area tedesca si è avuta l'inconsueta possibilità di rimpiazzare in toto la classe dirigente compromessa con il passato. Propio per questo si è potuto ricostruire prima l'identikit del democratico da manuale, per poi considerare legati al regime tutti coloro i quali presentavano un curriculum non perfettamente coincidente con quell'immagine. ${ }^{9}$

Peraltro le continuità non sono solo personali, e non riguardano i soli cultori del diritto: vi sono anche continuità istituzionali che caratterizzano il passaggio dalla dittatura alla democrazia, e continuità personali che interessano anche e soprattutto coloro i quali hanno animato la vita economica.
5 Sulla scia di un grande classico: KaRL Polanyi, The Great Transformation: The Political and Economic Origins of Our Time (1944), 2. ed., Boston 2002.

6 Wolfgang Wippermann, Totalitarismustheorien. Die Entwicklung der Diskussion von den Anfängen bis heute, Darmstadt 1997.
7 Angus Maddison, La natura e il funzionamento del capitalismo europeo, in: Moneta e Credito 51 (1988) $72-78$.

8 Citazioni in Alessandro Somma, I giuristi e l'Asse culturale RomaBerlino. Economia e politica nel diritto fascista e nazionalsocialista, Frankfurt am Main 2005, 4-22.
9 Cfr. Inga Markowits, Die Abwicklung. Ein Tagebuch zum Ende der DDR-Justiz, München 1993. 
E la prospettiva di Schröder, le cui analisi si muovono a un livello molto astratto, non è certo capace di incrementare le conoscenze relative a questi aspetti. È sicuramente importante mettere in luce le analogie che caratterizzano la metodologia giuridica e la teoria delle fonti diffuse nelle dittature tedesche, ma altrettanto sicuramente questo dato dice poco o nulla se dissociato dal contenuto delle regole maneggiate dai giuristi. Tanto più che ai giuristi è oramai nota la circostanza per cui non vi è corrispondenza univoca tra le tecniche impiegate e i valori di volta in volta veicolati, ovvero che sul piano fattuale vi possono essere equivalenze funzionali tra costruzioni anche radicalmente differenti.

Insomma, se del regime nazista e della Repubblica democratica tedesca si dice che si sono edificati entrambi su sistemi di regole destinati a imporre la volontà del Führer e del Partito unico, allora l'insieme dei centri di interesse radicati nell'apparato statale, o nel sistema produttivo, o nell'esercito o ancora nel mondo della formazione e dell'informazione finisce per essere privo di contorni definiti, se non addirittura per sparire dalla scena. Il che rappresenta, come abbiamo detto, il risvolto naturale delle teorie sul totalitarismo applicate a vicende ulteriori rispetto a quelle direttamente relative alla compressione delle libertà politiche.

Nazismo e postdiritto - Le teorie sul totalitarismo, per quanto siano state rivisitate e completate nel corso degli anni, restano dunque inadatte a mettere in luce la circostanza che il fascismo è stato un ordine politico, ma anche se non soprattutto un ordine economico: che vi è stata un'economia nazista fondata su un diritto nazista, i cui tratti si possono evidenziare ricorrendo alla teorie sul fascismo, di cui è agevole mettere in luce notevoli punti di contatto con le esperienze precedente e successiva. E di cui è possibile invece marcare le differenze rispetto all'economia socialista e al relativo diritto socialista, tanto che, almeno in questo ambito, qualsiasi raffronto manca clamorosamente l'obiettivo di contribuire a incrementare le nostre conoscenze sul totalitarismo in quanto punto di riferimento per la classificazione delle dittature.
Ovviamente qui non si tratta di relativizzare il giudizio sul nazismo, bensì di mettere in guardia rispetto alla possibilità che si riproponga nell'ordine economico, piuttosto che nell'ordine politico, con il rischio sempre in agguato che anche quest'ultimo finisca prima o poi per regredire. È del resto noto che i fondamenti del neoliberalismo si definiscono nel corso degli anni Trenta con il contributo fondamentale di studiosi compromessi con il nazismo, e che poi confluiscono in teorie e pratiche di governo dell'ordine economico adottate a partire dalla conclusione del secondo conflitto mondiale. ${ }^{\mathbf{1 0}}$ Teorie e pratiche che non sono di per sé ostili alla democrazia, ma che però sono insensibili alle sue sorti nella misura in cui ammettono una compressione delle libertà politiche ove richiesta per sostenere il sistema delle libertà economiche.

Le ricerche di Schröder non rappresentano un contributo utile a decodificare eventuali trasformazioni nel senso indicato, e non solo per il loro essere legate a doppio filo alle teorie sul totalitarismo. Problematica è anche la relativa caricatura dell'ordine liberale e democratico, definito come l'ordine nel quale il diritto si identifica a partire da concezioni volontaristiche, l'interpretazione del diritto può rifarsi a teorie oggettive ma non anche a sistemi di valori imposti dal potere politico, e la scienza giuridica valorizza la finalità delle regole.

Ebbene, il neoliberalismo affida ai pubblici poteri il compito di presidiare il mercato traducendo le sue leggi in leggi dello Stato, e così facendo impone mutamenti epocali quanto al metodo e all'oggetto della scienza giuridica. Non si tratta più di individuare e maneggiare regole a partire da un patrimonio sapienziale proprio del giurista: tanto da rappresentare il fondamento della sua legittimazione in quanto tecnocrate. Gli strumenti indispensabili a trovare e comprendere le regole sono oramai quelli mutuati dalla scienza economica, e in particolare dai paradigmi ora prevalenti: quelli contemplati dall'ortodossia neoliberale.

Viviamo cioè nell'epoca del postdiritto, espressione nella quale il prefisso "post « indica la negazione di ciò cui allude la parola alla quale viene anteposto, ovvero che il diritto si riduce oramai a 
mero riflesso di altri fenomeni sociali. E lo stesso accade a coloro i quali pretendono, per il tramite del diritto, di disciplinare lo stare insieme come società: i cultori del postdiritto sono i detentori di un sapere oramai incapace di interpretare $i$ fenomeni sociali, e tanto meno di governarli. ${ }^{\mathbf{1 1}} \mathrm{E}$ se ciò è avvenuto per quanto attiene al contenuto delle regole, non significa che non ci siano trasformazioni circa il modo di accertarle e di intenderle, e soprattutto che non si debba predisporsi a cogliere il nesso tra i due profili.

11 Cfr. Alessandro Somma, Verso il postdiritto? Fine della storia e spoliticizzazione dell'ordine economico, in: Politica del diritto 59 (2018) 79-109. 